\title{
Indicadores para valorar el grado de significación de los aprendizajes en las asignaturas de formación profesional
}

\section{Indicators to assess the degree of significance of the learning in the subjects of vocational training}

Virgilio Lucas Ramos Rivero

Universidad Internacional del Ecuador, Ecuador

Raúl Alvino Pesantes Rendón

Universidad Internacional del Ecuador, Ecuador

Autor para correspondencia: virgiliolucas@gmail.com

Fecha de recepción: 18 de Julio de 2016 - Fecha de aceptación: 30 de Agosto de 2016

\section{Resumen}

Este estudio presenta la importancia de la valoración del grado de significación de los aprendizajes en el contexto de las asignaturas de formación profesional en la carrera de Ingeniería en Mecánica Automotriz. En el mismo se fundamenta que una adecuada y cuidadosa labor del docente a la hora de determinar los indicadores, permitirá a este, llegar a resultados más objetivos en la evaluación de la construcción de significados logrados por el estudiante.

Palabras claves: grado de significación de los aprendizajes; indicadores de valoración; evaluación

\begin{abstract}
This study presents the importance of assessing the degree of significance of the learning in the context of the vocational subjects in Mechanical Engineering in Automotive. It is based on that an adequate and careful work of teachers when determining the indicators will allow them to achieve results more objective in assessing the construction of the meaning achieved by the student.
\end{abstract}

Key words: significance of learning; valuation indicators; evaluation 


\section{Introducción}

Un tema de singular importancia en el ámbito de la docencia, es sin duda el relacionado con el aprendizaje significativo, acerca del cual existe una amplia y variada bibliografía. No obstante, si importante resulta tener un conocimiento del tema, más importante aún es contar con las herramientas didácticas y medios que permitan evidenciar que se ha logrado un verdadero aprendizaje significativo, así como en qué grado o medida este puede considerarse satisfactorio o insuficiente.

Partiendo de diferentes fuentes de información y de la propia experiencia de los autores, en el presente artículo se trata el tema relacionado con el grado de significación del aprendizaje y se proponen algunos indicadores que derivados de la funcionalidad de los contenidos que se aspira lograr en los estudiantes, deben contribuir a valorar de forma más objetiva dicha significación.

Los indicadores que se proponen van dirigidos a la valoración del grado de significación de los aprendizajes en las asignaturas de formación profesional de la Carrera de Ingeniería en Mecánica Automotriz que se desarrolla en la Universidad Internacional del Ecuador (UIDE), Extensión Guayaquil.

Aunque en el artículo no se trata en toda su amplitud el tema de la evaluación de los aprendizajes, si evidencia su estrecho vínculo con el mismo mediante algunas reflexiones y deja sentadas algunas pautas para un estudio posterior.

\section{Materiales y métodos}

\section{Aprendizaje significativo}

El aprendizaje significativo es parte consustancial del enfoque constructivista del aprendizaje y fue planteado por Ausubel en oposición a los aprendizajes obtenidos por los estudiantes como resultado de la enseñanza tradicional.

De acuerdo con la teoría del aprendizaje significativo, para que este se produzca, se deben cumplir determinadas condiciones relativas al material objeto de estudio y al estudiante.

Para Ausubel (1997) la consecución de aprendizajes significativos depende de dos condiciones:

1. Que el material objeto de aprendizaje sea potencialmente significativo, lo cual significa que el mismo debe estar organizado de modo que se relacione con la estructura cognitiva del estudiante y que aparezca en su mente como organizado.

2. Que el que aprende tenga la disposición de hacerlo significativamente

La primera condición debe inducir al docente a organizar rigurosamente el material presentado al estudiante y a diagnosticar objetivamente si el discente cuenta con los 
prerrequisitos para asimilar el nuevo contenido. La segunda condición está vinculada con la motivación, de modo que el aprendizaje no se producirá significativamente si el sujeto de aprendizaje no muestra interés en hacerlo.

Pimienta (2007) señala además como otra condición para el aprendizaje significativo, la actitud activa y motivadora que adopte el docente durante el proceso de aprendizaje de los estudiantes, teniendo en cuenta el papel de facilitador o mediador que debe jugar el mismo.

Es importante establecer la diferencia entre el aprendizaje significativo y el significado de lo que se aprende. El aprendizaje significativo se refleja en el proceso de asimilación de los contenidos por el estudiante bajo la guía del docente así como en los resultados de dicho proceso de asimilación. Por su parte, el significado de lo aprendido está en relación con los contenidos de la asignatura de que se trate, así por ejemplo lo que puede ser significativo para la Matemática como ciencia, no tiene que ser significativo para la Física o para asignaturas de formación profesional de la Carrera de Ingeniería Mecánica Automotriz, tales como: Tecnología de materiales, Combustibles y lubricantes, Soldadura, Maquinaria para la reconstrucción de motores entre otras.

Los resultados de los aprendizajes serán la evidencia consustancial que permitirá valorar, el grado de significación de lo aprendido por los estudiantes.

Ahora bien, ¿Qué es el grado de significación de los aprendizajes?

\section{Grado de significación de los aprendizajes}

Determinar si el aprendizaje de los estudiantes ha sido significativo o no y en qué medida, es una tarea de crucial importancia para el docente y en este aspecto, la evaluación se constituye en la principal herramienta.

La evaluación realizada en el contexto de una asignatura, debe validar, medir, constatar, comprobar aprendizajes significativos y en qué grado se han alcanzado (grado de significancia o de significado).

Es por demás muy importante dar un criterio acerca del grado de significatividad alcanzado por el estudiante, a fin de tener con la mayor certeza posible, el nivel de asimilación, complejidad y amplitud de los aprendizajes logrados.

Díaz (2002) reconoce que no es sencillo valorar la significatividad del aprendizaje alcanzada por el estudiante teniendo en cuenta que el aprendizaje significativo se obtiene gradualmente y que su valoración es cualitativa además de que requiere el empleo de indicadores. Estos deben precisar qué se quiere obtener como resultado del aprendizaje y reflejar si los estudiantes han cumplido o no de forma satisfactoria los requerimientos de la asignatura de que se trate. De no precisar adecuadamente dichos indicadores, la evaluación no sería verdaderamente objetiva (en correspondencia con la realidad). 
Lo anterior implica determinar según Díaz (2002) el nivel de comprensión alcanzado por los estudiantes en relación a los contenidos estudiados.

Este nivel de comprensión se debe reflejar en los resultados de aprendizaje que son, las transformaciones que se hayan producido en el estudiante, en cuanto a los niveles de conocimiento, comprensión y competencias, como consecuencia del proceso de aprendizaje (Ibarra y Rodríguez, 2011).

Los indicadores como expresa Cerda (2000) son los que facilitan la operacionalización de los resultados del aprendizaje, análogamente a como permiten operacionalizar las variables en una investigación científica.

La significatividad de los aprendizajes Coll, (1996), tiene un estrecho vínculo con su funcionalidad, lo cual quiere decir, que los conocimientos, habilidades, valores, actitudes y normas, puedan ser efectivamente utilizadas bajo cualquier condición por los estudiantes.

Las posibilidades de utilización de dichos contenidos y por ende, la variedad y complejidad de los problemas relacionados con la profesión que serán capaces de resolver los estudiantes de una carrera técnica, estarán así mismo relacionados con la amplitud y complejidad de las redes cognitivas formadas.

Según Coll (1996), a mayor número y complejidad de las relaciones entre el nuevo material de aprendizaje y el que está presente en la estructura cognitiva del estudiante, mayor será el grado de significación del aprendizaje y tanto mayor será también su funcionalidad. La misma relación existe entre el nivel de asimilación del material estudiado y el grado de significación del aprendizaje, entendiendo por nivel de asimilación el grado de dominio de los contenidos, es decir, la apropiación completa del conocimiento y de las habilidades vinculadas a dicho conocimiento en determinadas condiciones (Álvarez, 1988).

A lo anterior, al tratar del nivel de asimilación de una competencia, habría que incluir dentro del grado de dominio de la competencia, los valores manifiestos en el estudiante en su actuación profesional.

La funcionalidad puede considerarse como una dimensión del grado de significación del aprendizaje, mientras que la amplitud y la complejidad del material de estudio pueden considerarse indicadores de la funcionalidad (Ramos, 2015).

Por otro lado, los indicadores de la funcionalidad, deben estar íntimamente relacionadas con las características de la asignatura de que se trate.

Es importante destacar que la variedad y complejidad creciente reflejan el nivel de integración alcanzado con el material de estudio, es decir, que el aprendizaje debe realizarse como un proceso de integración e interrelación en orden creciente y globalizado del contenido, que contribuya a formar estructuras cognitivas cada vez más complejas, lo que aumentará las capacidades resolutivas del estudiante. 
Otro elemento consustancial de la funcionalidad es la flexibilidad con que se puede hacer uso de lo aprendido.

En relación a esto Díaz y Hernández (2003) consideran la flexibilidad de la aplicación de lo aprendido como un componente central de la funcionalidad, de igual forma que la variedad y complejidad crecientes.

La flexibilidad, como indicador de la funcionalidad, es otra característica importante del contenido aprendido por el estudiante en la carrera. Dicho contenido debe estar organizado alrededor de núcleos de contenidos, que, por su nivel de generalización, puedan ser aplicados a la solución de diversos problemas propios de la profesión ingenieril.

Vázquez y Alarcón (2010), señalan que la significatividad se induce a través de indicadores tales como la estabilidad y durabilidad en el tiempo de lo aprendido y su transferibilidad, es decir, la capacidad de aplicación del aprendizaje en otros contextos diferentes de aquel donde se aprendió.

A juicio de los autores, el grado de significación del aprendizaje es una variable que expresa la medida en que el discente ha atribuido un significado al contenido asimilado, en relación a los objetivos del nivel de sistematicidad de los contenidos de que se trate, es decir, dependiendo de si se trata del nivel de carrera, asignatura, unidad de aprendizaje o tema de una clase en particular.

Valorando los elementos de carácter teórico que se manejan en la literatura consultada en relacionada al grado de significación de los aprendizajes y contextualizándolo al nivel de una asignatura, podemos conceptualizar dicha variable como:

Medida en que los estudiantes han sido capaces de construir con sus propios recursos cognitivos y la mediación del docente, interpretaciones significativas y valiosas de los contenidos estudiados y atribuir un valor funcional a dichas interpretaciones en función de las competencias previstas en el micro currículo de la asignatura cursada.

\section{Resultados y discusiones}

\section{Las asignaturas de formación profesional de la carrera de Ingeniería en Mecánica Automotriz}

Las asignaturas de formación profesional, tienen como propósito el desarrollo de competencias en el estudiante relacionadas directamente con el ejercicio de la profesión (Ramos, 2015).

En el diseño curricular de la Carrera de Ingenieria en Mecánica Automotriz de la UIDE está definido el eje de formación profesional que incluye todas las asignaturas del ejercicio de la profesión de la mencionada carrera, El dominio de las competencias contempladas en los micro currículos de dichas asignaturas permite a los estudiantes resolver un gran número de problemas 
de diferente naturaleza, amplitud y complejidad, que tributan a la solución de problemas superiores hasta alcanzar el perfil de la profesión.

Ahora bien, ¿Cómo podemos saber si lo aprendido es significativo o no en el contexto de una asignatura de formación profesional?

\section{Dimensiones e indicadores del grado de significación de los aprendizajes.}

Teniendo en cuenta que los resultados de la evaluación de las competencias, deben expresar el grado de significación de los aprendizajes logrados por los estudiantes en relación con estas, es decir, el dominio de las competencias objeto de evaluación, la precisión de dimensiones e indicadores para valorar dicha significación resulta por demás indispensable.

Según la definición de Grado de significación de los aprendizajes, la funcionalidad es uno de las dimensiones más potentes para realizar su valoración, entendiendo la funcionalidad como la dimensión del grado de significación de los aprendizajes que permite valorar si los conocimientos, habilidades, destrezas, valores y actitudes aprendidos por los estudiantes, pueden ser efectivamente utilizados por este en la solución de problemas de la profesión y en la construcción de nuevos significados.

Partiendo de las características de las asignaturas de formación profesional de la carrera de Ingeniería en Mecánica automotriz que se desarrolla en la UIDE. Extensión Guayaquil y teniendo en cuenta la experiencia de los autores en los procesos evaluativos de las referidas asignaturas, a continuación, se presenta una tabla con la dimensión funcionalidad y algunos indicadores que se proponen para su verificación en el proceso evaluativo en una asignatura de formación profesional de la carrera de Ingeniería en Mecánica Automotriz.

Tabla $\mathrm{N}^{\circ} 1$. Indicadores para determinar el grado de significación

\begin{tabular}{ll}
\hline Dimensión & \multicolumn{1}{c}{ Indicadores } \\
\cline { 2 - 3 } & \multicolumn{1}{c}{ Diseño de mapas conceptuales } \\
Modelación y solución de problemas de la profesión \\
Nivel de amplitud de los contenidos evaluados \\
Nivel de complejidad de las tareas solucionadas \\
Habilidad para el manejo de equipos, herramientas e instrumentos \\
Dominio de procesos tecnológicos \\
Dominio de estructuras componentes y funciones de sistemas tecnológicos \\
\\
Duncionalidad \\
Solución creativa de problemas tecnológicos \\
Integración de contenidos \\
Construcción de nuevos aprendizajes \\
Demostración de valores y actitudes deseables \\
& Flexibilidad en la aplicación de los contenidos
\end{tabular}

Fuente: Autor 
El docente en el ejercicio de su actividad académica, podrá adecuar los indicadores anteriores o definir otros indicadores de la funcionalidad teniendo en cuenta las características de la asignatura del ejercicio de la profesión que orienta, e incluso determinar otras dimensiones para valorar el grado de significación de los aprendizajes.

Para llegar a conclusiones objetivas acerca del grado de significación de los aprendizajes alcanzado por los estudiantes, es necesaria una cuidadosa labor del docente a la hora de determinar los indicadores para evaluar los contenidos de carácter conceptual, procedimental y actitudinal objeto de aprendizaje y establecer los criterios de valoración del grado o nivel alcanzado por el estudiante. Por otro lado, el adecuado diseño de los instrumentos de evaluación y la elaboración de las rúbricas correspondientes, son herramientas de gran utilidad para poder cumplir con este propósito.

\section{Conclusiones y recomendaciones}

La certeza o no del logro de un aprendizaje significativo puede tenerse a partir del desempeño de los estudiantes y la medida o grado de significación de ese logro.

Para que el docente pueda realizar una valoración adecuada de la significación de lo aprendido por el estudiante, debe en primer lugar, tener una clara comprensión de lo que significa el grado de significación de los aprendizajes y en segundo lugar, tendrá que asumir la tarea realmente compleja de establecer los indicadores necesarios que permitan objetivizar el principio de la significatividad y la funcionalidad de los aprendizajes (Coll, 1994), teniendo en cuenta las características y alcances de la asignatura de formación profesional de que se trate.

Existe una estrecha relación entre la evaluación del proceso de aprendizaje del estudiante y el grado de significación de lo aprendido conceptual, procedimental y actitudinalmente por este.

\section{Bibliografía}

Álvarez, Z. (1988). Fundamentos teóricos de la dirección del proceso de formación profesional de perfil amplio. Villa Clara: Universidad Central de las Villas.

Ausubel, D., Novak, J. y Hanesian, H. (1997). Psicología Educativa. Un punto de vista cognoscitivo. México, Trillas.

Barriga Arceo, F., \& Hernández Rojas, G. (2002). Estrategias docentes para un aprendizaje significativo: Una interpretación constructivista (Segunda ed.). México: McGraw-Hill Interamericana.

Cerda, H. (2003). La evaluación como experiencia total. Logros -objetivos -procesos competencias y desempeño. Colombia: Editorial Delfin Ltda.

Coll, C. (1996). Aprendizaje escolar y construcción del conocimiento. Buenos Aires: Paidós Educador. 
Pimienta, J. (2007). Metodología constructivista (Segunda ed.). México: Pearson Educación.

Ramos, R. (2015). Incidencia de la evaluación de las asignaturas de formación profesional en la valoración del grado de significación de los aprendizajes de los estudiantes de la carrera de Ingeniería Mecánica Automotriz de la UIDE, Extensión Guayaquil, 2013 - 2014. Diseño de un manual práctico de evaluación integral de los estudiantes (tesis de maestría). Universidad de Guayaquil, Ecuador.

Rodríguez, G., \& Ibarra, S. (2011). Evaluación orientada al aprendizaje estratégico. Madrid: Narcea, S.A.

Vázquez, Á., \& Alarcón, M. (2010). Didáctica de la Tecnología. Madrid: SINTESIS 